\title{
Higher gestational weight gain and lower serum estradiol levels on the day of human chorionic gonadotropin administration are associated with increased risk of preeclampsia after in vitro fertilization
}

\section{Yu-Chen Chen}

Chang Gung Memorial Hospital Kaohsiung Branch

\section{Yun-Ju Lai}

Chang Gung Memorial Hospital Kaohsiung Branch

\section{Yu-Ting Su}

Chang Gung Memorial Hospital Kaohsiung Branch

\section{Ni-Chin Tsai}

Chang Gung Memorial Hospital Kaohsiung Branch

Kuo-Chung Lan ( $\sim$ lankuochung@gmail.com )

Department of Obstetrics and Gyneclogy ,Kaohsiung Chang Gung Memorial Hospital and Chang Gung University college of Medicine ,Kaohsiung ,Taiwan https://orcid.org/0000-0003-3702-0091

\section{Research}

Keywords: Estradiol (E2), Progesterone (P4), controlled ovarian hyperstimulation (COH), body weight, preeclampsia

Posted Date: January 21st, 2020

DOl: https://doi.org/10.21203/rs.2.21456/v1

License: (c) (i) This work is licensed under a Creative Commons Attribution 4.0 International License. Read Full License 


\section{Abstract}

Background Very few previous studies have examined the effect of endocrine parameters during ART on preeclampsia. Moreover, there is little known about the relationship of steroid hormone levels on development of the placenta. The purpose of this study is to assess the association of preeclampsia with serum estradiol (E2) and progesterone (P4) levels on the day of human chorionic gonadotropin (hCG) administration during controlled ovarian hyperstimulation $(\mathrm{COH})$ for in vitro fertilization/intracytoplasmic sperm injection (IVF/ICSI).

Methods This was a hospital-based cohort study using clinical data from the Kaohsiung Chang Gung Memorial Hospital Obstetric and Neonatal Database (KCGMHOND) from Jan 1, 2001 to December 1, 2018. Eligible women underwent at least one autologous IVF/ICSI cycle and had a live-born infant with a gestational age (GA) of more than 20 weeks.

Results A total of 622 women who had live births after fresh IVF/ICSI-ET during the study period met our inclusion criteria. Twenty-eight women (4.5\%) met the diagnostic criteria for preeclampsia. However, women in the preeclampsia group had a significantly higher body mass index ( $22 \mathrm{vs.} 24, p=0.05)$, body weight at delivery (70.0 vs. $80.5 \mathrm{~kg}, \mathrm{p}<0.001)$ and gestational weight gain (13.0 vs. $19.6 \mathrm{~kg}, \mathrm{p}=0.002)$ and had lower use of ICSI $(29.9 \%$ vs. $10.7 \%, p=0.021)$. Logistic regression analysis of the relationship of patient and treatment characteristics with preeclampsia. The crude ORs indicated that young female age $>34$, not using ICSI, E2 peak $<1200 \mathrm{pg} / \mathrm{mL}$ and gestational weight gain $>20 \mathrm{~kg}$ were associated with preeclampsia. After adjustment for confounding, the only factors that remained significant were E2 peak $<1200 \mathrm{pg} / \mathrm{mL}(\mathrm{aOR}=4.634,95 \% \mathrm{Cl}=1.061$ to 20.222$)$, and gestational weight gain $>20 \mathrm{~kg}$ (aOR: 13.601 , $95 \%$ Cl: 3.784, 48.880).

Conclusions For women receiving IVF/ICSI, lower estradiol hormone levels on the day of hCG administration and higher pregnancy weight gain are related with subsequent preeclampsia.

\section{Background}

Preeclampsia, a disorder characterized by hypertension and proteinuria during pregnancy, affects 5 to $8 \%$ of pregnant women worldwide and is a leading cause of obstetric complications [1]. In addition to hypertension and proteinuria, these patients also experience placental ischemia, endothelial dysfunction, end-organ ischemia, and vascular complications [1-3].

Many studies have examined the pathogeneses of these two conditions, although the exact mechanism(s) remain unknown [2, 4]. However, research has established that the pathogenesis of preeclampsia is characterized by aberrant trophoblastic invasion of the decidual and myometrial spiral arteries during early pregnancy, and that this leads to placental dysfunction and ischemia $[5,6]$.

Alterations in the syntheses and interactions of multiple steroidal hormones, including androgens, estrogens, and progestogens, occurs in the gonads and placenta during early pregnancy. These 
hormones are important in regulating the growth and differentiation of the placental trophoblast and the placental vascular tree, and in promoting extravillous cytotrophoblast invasion of the uterine endometrium and myometrium [1]. Thus, previous studies of the mechanism of preeclampsia have compared the levels of steroid hormones and hormone signaling in pregnant women with and without preeclampsia [7-10].

The effect of different modalities of assisted reproductive technology (ART) on adverse pregnancy outcomes, including hypertensive complications and preeclampsia, are unknown [11-14]. During ART procedures, many endocrine parameters could potentially have an adverse impact on implantation or placentation $[15,16]$. It is possible that an aberrant endocrine profile (especially the levels of estrogens or progestogens) causes the placenta to be abnormal, or alternatively that an abnormal placenta causes aberrant production of estrogens and progestogens. Very few previous studies have examined the effect of endocrine parameters during ART on preeclampsia. Moreover, there is little known about the relationship of steroid hormone levels on development of the placenta. Only two studies thus lead to the hypothesis that abnormal placentation is mediated by an elevated E2 level at the time of implantation, because this adversely affects trophoblastic invasion of the endometrium[17, 18]. However, multiple studies reported an increased risk of preeclampsia in women conceiving by IVF with a frozen ET[19-23]. From this point of view, high levels of steroid hormones (E2 and P4) do not appear to be the main cause of placental dysplasia during implantation. Instead, these steroid hormones should be considered to play an ancillary role placental dysplasia.

In this study of women receiving ART procedures, we examined whether estradiol (E2) and progesterone (P4) levels on the day of human chorionic gonadotropin ( $\mathrm{hCG}$ ) administration were associated with subsequent preeclampsia. We also aimed to identify with the major factors associated of preeclampsia outcomes in women undergoing IVF/ICSI procedures.

\section{Materials And Methods}

\section{Study Design}

This was a hospital-based cohort study using clinical data from the Kaohsiung Chang Gung Memorial Hospital Obstetric and Neonatal Database (KCGMHOND) from Jan 1, 2001 to December 1, 2018. KCGMHOND records all live births and stillbirths weighing at least 500 grams and pregnancies delivered after 20 weeks gestation (calculated using date of last normal menstrual period, if available, or estimated date of confinement by ultrasound otherwise) at a tertiary university hospital in Kaohsiung, Taiwan. The maternal age, parity, birth weight, gestational age (GA) at delivery, mode of delivery, and sex were recorded for all live births. We supplemented prenatal data and medical comorbidities including chronic hypertension, diabetes mellitus, renal disease, hypothyroidism from KCGMH prenatal clinic charts matched to the index pregnancy in KCGMHOND. Furthermore, we have data on prior events including a history of preeclampsia among multiparous women. 
The reproductive clinic data were linked with KCGMHOND using unique patient identifiers as well as the date and time of delivery associated with the fresh IVF/ICSI fertility treatment procedures, and the following data were collected: age, body mass index (BMI), E2 and P4 levels on the day of the hCG trigger, infertility diagnosis, number of embryos transferred, duration of ovarian stimulation, total gonadotropins administered, peak endometrial thickness, total number of retrieved mature oocytes, and use of blastocyst-stage transfer. The study was conducted following approval of Ethics Committee of Institutional Review Board (CGMH 201901565B0). The presence of preeclampsia was defined by an elevated systolic blood pressure $(>140 \mathrm{mmHg}$ ) or diastolic blood pressure ( $>90 \mathrm{mmHg}$ ) after 20 weeks of gestation and with proteinuria or other clinical signs and symptoms. Intrauterine growth restriction (IUGR) was defined as an estimated fetal weight below the 10th percentile. Women were excluded of they had utilizing donor oocytes.

\section{Assisted reproductive technologies}

Our previous research described the methods used for controlled ovarian stimulation (COS), oocyte retrieval, embryo culture, and embryo transfer $(\mathrm{ET})[16,24]$. The long protocol and the $\mathrm{GnRH}$-ant protocol for COS were individualized according to each patient's ovarian reserve, age, baseline level of serum follicle stimulating hormone (FSH), and prior response to COS.

The long protocol consisted of administration of leuprolide acetate (Lupron®; Takeda, Tokyo, Japan) with the initial dose of gonadotropin (human menopausal gonadotropin [hMG] or purified recombinant FSH) at a dose of 150 to 300 IU. Dose adjustments were based on each patient's ovarian response and according to the serum E2 level and follicular growth (determined by ultrasonography). When the lead follicle had a diameter of 17 to $19 \mathrm{~mm}$, leuprolide acetate and FSH were halted, and hCG (Ovidrel ${ }^{\circledR}$; Serono, Modugno, Italy) administration began. Thirty-six to $38 \mathrm{~h}$ later, oocyte retrieval was performed using transvaginal ultrasound-guided follicle aspiration.

The flexible $\mathrm{GnRH}$-ant protocol consisted of gonadotropin stimulation and then suppression $(0.25 \mathrm{mg}$ ganirelix acetate, MSD; $0.25 \mathrm{mg}$ cetrorelix acetate, Serono) when the lead follicle had a diameter of $14 \mathrm{~mm}$. hCG (6500 IU, Ovidrel ${ }^{\circledR}$, Serono, Modugno, Italy) was administered when 2 more follicles had diameters of $17 \mathrm{~mm}$. Oocyte retrieval was performed as described above.

Oocyte maturity was measured by analysis of the cumulus mass, corona radiata, ooplasm, and detachment of membrane granulosa cells. IVF/ICSI was performed as previously described [24], and fertilization was assessed 16 to $18 \mathrm{~h}$ later. Successful fertilization was defined by the presence of a zygote with two pronuclei. One team of embryologists oversaw all laboratory procedures to ensure standardization.

\section{Statistical analysis}

Categorical variables are expressed as percentages and continuous variables as medians (interquartile ranges [IQRs]) or means \pm standard deviations (SDs), as appropriate. The Shapiro-Wilk normality test was used to check for normality of distributions. Mann-Whitney rank-sum test for the comparison of medians. 
Receiver operating characteristic (ROC) curve was used to determine optimal cutoff of dosimetric data for preeclampsia. After determination of optimal cutoff using ROC curve, logistic regression model using categorial variable was used for confirmation of dosimetric significance. Multivariable logistic regression analysis was used to assess the relationship of preeclampsia with endocrine parameters, mother's age, age of the male partner, BMI, infertility diagnosis, ovarian stimulation protocol, duration of ovarian stimulation, maximal endometrial thickness, number oocytes retrieved, number of embryos transferred, use of ICSI, use of blastocyst-stage ET, occurrence of multiple pregnancies, and pregnancy weight gain. A full model, with the inclusion of all variables to adequately control for potential confounding, was implemented and the results are presented as adjusted odds ratios (aORs) and 95\% confidence interval ( $95 \% \mathrm{Cls}$ ). An OR was considered statistically significant if its two-tailed $p$-value was less than 0.05 or if its $95 \% \mathrm{Cl}$ did not include one. A P-value below 0.05 was considered significant, and all statistical analyses were performed using SPSS for Windows (version 20).

\section{Results}

A total of 622 women who had live births after fresh IVF/ICSI-ET during the study period met our inclusion criteria (Table 1). Twenty-eight women (4.5\%) met the diagnostic criteria for preeclampsia. Comparison of the baseline characteristics of women with and without preeclampsia indicated no significant difference in age (34.0 vs. 35.0 years), age of the male partner (36.0 vs. 37.5 years), duration of stimulation ( 9.0 vs. 9.0 days), total gonadotropin administered ( 29.0 vs. 31.0 ampoules), endometrium thickness ( 1.3 vs. $1.3 \mathrm{~cm}$ ), number of mature oocytes retrieved (7.0 vs. 7.0), number of embryos transferred (2.0 vs. 2.0), use of blastocyst transfer (63.1\% vs. $64.3 \%)$, E2 level on the day of hCG administration (1802 vs. $1759 \mathrm{pg} / \mathrm{mL}$ ) and P4 level on the day of hCG administration (1.0 vs. $1.2 \mathrm{ng} / \mathrm{mL}$ ). However, women with preeclampsia had a significantly higher body mass index ( $22 \mathrm{vs.24,p}=0.05$ ), body weight at delivery $(70.0$ vs.80.5 kg, $p<0.001)$ and gestational weight gain $(13.0 \mathrm{vs} .19 .6 \mathrm{~kg}, \mathrm{p}=0.002)$ and had lower use of ICSI $(29.9 \%$ vs. $10.7 \%, p=0.021)$.

We also analyzed the obstetric and perinatal outcomes of the two groups (Table 2). There were no significant differences in the incidences of multiple pregnancy $(31.9 \%$ vs. $32.1 \%)$ or IUGR $(27.8 \%$ vs. $32.1 \%$ ) or sexual ratio; Birth weight (3100 vs. $2910 \mathrm{~g}$ ) of singletons; and GA at delivery ( $36 \mathrm{vs.} 36$ weeks) and birth weight ( 2370 vs. $2420 \mathrm{~g}$ ) of twins. However, women in the preeclampsia group had significantly higher incidence of Caesarean section ( $53.5 \%$ vs. $82.1 \%, p<0.001)$ and lower GA at delivery (38 vs. 36 weeks, $p<0.001)$ age at singleton.

Logistic regression analysis of the relationship of patient and treatment characteristics with preeclampsia. The crude ORs indicated that young female age $>34$, not using ICSI, E2 peak $<1200 \mathrm{pg} / \mathrm{mL}$ and gestational weight gain $>20 \mathrm{~kg}$ were associated with preeclampsia. After adjustment for confounding, the only factors that remained significant were E2 peak $<1200 \mathrm{pg} / \mathrm{mL}(\mathrm{aOR}=4.634,95 \% \mathrm{Cl}$ $=1.061$ to 20.222 ), and gestational weight gain $>20 \mathrm{~kg}$ (aOR: 13.601, 95\% Cl: $3.784,48.880)$. (Table 3 ).

\section{Discussion}


We compared women from a single institution over a 18-year period who did or did not develop preeclampsia following an ART procedure. Our univariate analysis indicated that women who developed preeclampsia had a lower E2 level and higher gestational weight gain and lower using ICSI. However, after adjustment for confounding factors, our multivariate analysis indicated that lower E2 and higher gestational weight gain related to preeclampsia. Moreover, women in the two groups had similar incidences of IUGR.

There is evidence that dysregulation of E2 production in the placenta is associated with preeclampsia, thus suggesting that the E2 level could be used as a biomarker of preeclampsia and that its clinical modulation could prevent or cure preeclampsia [10]. In fact, it is well-recognized that aberrant production of estrogens by the placenta plays a key role in preeclampsia symptoms, because these hormones promote angiogenesis and vasodilation [10]. Indeed, previous studies have documented low plasma E2 concentrations in women with severe $[9,25]$ and mild [26] preeclampsia, and low levels in the placental tissues of women with preeclampsia [27]. P4 modulates the synthesis and release of angiogenic factors by placental cells, and regulates trophoblastic invasion and uterine artery remodeling. Research has also indicated the presence of lower P4 levels in women with preeclampsia compared with healthy pregnant women $[8,28-30]$.

However, few studies have evaluated the role of E2 and P4 in preeclampsia during the first trimester, especially before the luteo-placental shift or the embryo implantation stage [31]. A previous study of baboons reported that elevation of E2 levels during the first trimester was associated with attenuation of extravillous trophoblast invasion of the uterine spiral arteries [32]. Imudia et al. reported that a high E2 level on the day of hCG administration during $\mathrm{COH}$ was associated with an increased risk for preeclampsia [17]. Another study of women who received ICSI found that an elevated E2 level on the day of hCG administration was associated with adverse obstetric outcomes related to alterations of placentation [18]. These studies thus lead to the hypothesis that abnormal placentation is mediated by an elevated E2 level at the time of implantation, because this adversely affects trophoblastic invasion of the endometrium. Thus, an elevated E2 peak during an ART procedure increases the risk of abnormal placentation, but has no significant effects on pregnancy, or miscarriage. Besides, premature P4 elevation on the day of HCG administration may cause an asynchronous endometrium, and negatively impact implantation and pregnancy [16]. A previous study reported that a high P4 level on the day of the hCG trigger was associated with preeclampsia, but that the E2 level had no such effect [14]. These findings thus differ from our results. Moreover, a recent publication also reported that the risk of preeclampsia was lower when there was a high peak E2 level [33].

Multiple studies reported an increased risk of preeclampsia in women conceiving by IVF with a frozen ET (FET) [19-23]. This may be related to the lack of a corpus luteum and the use of endometrial priming with estrogens during artificial FET cycles $[13,21]$. Indeed, during the thawing cycles, without the formation of a corpus luteum, the deficiency of relaxin may impair vasodilation and increase vascular dysfunction [11]. The corpus luteum is a temporary endocrine structure in female ovaries that produces high levels of P4 and moderate levels of E2 and inhibin A [34]. It is clear that serum P4 and E2 
concentrations after $\mathrm{COH}$ and ET are higher during early pregnancy (up to week-7 or week-8) than after FET [35]. From this point of view, high levels of steroid hormones (E2 and P4) do not appear to be the main cause of placental dysplasia during implantation. Instead, these steroid hormones should be considered to play an ancillary role placental dysplasia [11].

An ART procedure may induce epigenetic defects in the conceptus due to its modification of the embryo environment. Moreover, the hormone treatments during an ART procedure may impair endometrial receptivity. If the female lacks the physiological mechanisms for coping with these hormone injections, then improper maternal-fetal exchanges occur, potentially leading to preeclampsia or IUGR. Fortunately, in most cases, successful placental adaptation enables normal development and the birth of healthy offspring [36]. A recent systematic review concluded that every procedure used during high-technology infertility treatments can potentially increase the risk of an abnormal pregnancy and perinatal complications [37]. However, due to the inadequate quality of the current evidence, further well-designed studies are needed to address this issue.

There are two subtypes of preeclampsia - early-onset (before 34 weeks of gestation) and late-onset (after 34 weeks of gestation). Early-onset preeclampsia is associated with worse outcomes, and is believed to be caused by defective placentation. In contrast, late-onset preeclampsia is caused by interactions between the normal senescence of the placenta and a maternal genetic predisposition to cardiovascular and metabolic disease, and is not associated with IUGR $[2,38]$. Our results indicated that the incidence of IUGR were not significantly different in women with and without preeclampsia after $\mathrm{COH}$ and ET. Thus, the preeclampsia that occurs following IVF can be considered late-onset preeclampsia.

Metabolic abnormalities and obesity's impact on the risk for developing preeclampsia[39, 40]. Interestingly, not all obese pregnant women develop preeclampsia. Data suggest that obese pregnant women with the greatest metabolic abnormalities have the highest incidence of preeclampsia. Maternal adipokines, i.e., interleukin 6 (IL-6), tumor necrosis factor alpha (TNF-a), leptin and adiponectin link maternal nutritional status and adipose tissue metabolism to placental function[40]. High BMI is strongly associated with preeclampsia, and this risk is compounded in IVF pregnancies[41]. High pregnancy weight gain before diagnosis increases the risk of preeclampsia in nulliparous women and is more strongly associated with later-onset preeclampsia than early-onset preeclampsia[42, 43].

Our finding that the association between pregnancy weight gain and preeclampsia. It is worthy to conduct randomized trials assessing if interventions to reduce pregnancy weight gain decrease the risk of preeclampsia would be an appropriate next step in pregnancy women with lower E2 levels on the hCG day after IVF.

The present study had some limitations. Most of the patients were Taiwanese women, so it is necessary to be cautious when applying our results to other ethnic and racial groups. In addition, we could not exclude the presence of confounding due to socioeconomic status, because the delivery records at our facility lacked detailed information on household income. However, such a bias seems unlikely, because the women who undergo ART generally have higher than average incomes and education. Another 
limitation is that this was a retrospective study that examined women over a period of 18 years, during which time there were multiple practice changes, with a higher proportion of fresh ET cycles in the earlier part of the study. We could not account for all practice changes that might have influenced our results.

In conclusion, Higher gestational weigh gain and lower serum E2 levels on the day of hCG administration were associated preeclampsia in women who underwent an ART procedure. Women with and without preeclampsia had similar incidences of IUGR. Further studies are needed to determine the pathogenic mechanism of preeclampsia after IVF/ET.

\section{Abbreviations}

ART

assisted reproductive technology; BMI:body mass index; $\mathrm{COH}$ :controlled ovarian hyperstimulation; E2:estradiol; ET:embryo transfer; FSH:follicle stimulating hormone ; GnRH:gonadotrophin-releasing hormone; hCG:human chorionic gonadotropin; hMG:human menopausal gonadotropin;

ICSI:intracytoplasmic sperm injection; IL-6:interleukin 6 ; IUGR:intrauterine growth restriction; IVF:in vitro fertilization; KCGMHOND:Chang Gung Memorial Hospital Obstetric and Neonatal Database; ROC:Receiver operating characteristic; P4:progesterone; TNF-a:tumor necrosis factor alpha

\section{Declarations}

\section{Ethics approval}

This study was approved by the Institutional Review Board of Chang Gung Memorial Hospital (CGMH 201901565B0).

\section{Consent for publication}

Not applicable.

\section{Availability of data and materials}

The datasets used and/or analyzed during the current study are available from the corresponding author on reasonable request.

\section{Competing interests}

The authors declare that they have no competing interests.

\section{Funding}

This study was supported by CMRPG8G0071-73, CMRPG8H0981 from Chang Gung Memorial Hospital.

\section{Authors' contributions}


Conception and design of study: KCL; enrolled the subjects: YCC, YJL; analysis of data and writing of the manuscript: KCL,YCC,YJL; critical revision of the article for intellectual content: KCL YCC, YJL, YTS, NCT. All authors read and approved the final manuscript.

\section{Acknowledgments}

not applicable

\section{Author's information}

Department of Obstetrics and Gynecology, Kaohsiung Chang Gung Memorial Hospital and Chang Gung University College of Medicine, Kaohsiung, Taiwan

\section{References}

1.

Armaly Z, Jadaon JE, Jabbour A, Abassi ZA. Preeclampsia: Novel Mechanisms and Potential Therapeutic Approaches. Front Physiol. 2018;9:973.

2.

Burton GJ, Redman CW, Roberts JM, Moffett A. Pre-eclampsia: pathophysiology and clinical implications. BMJ. 2019;366:I2381.

3.

Wilkerson RG, Ogunbodede AC. Hypertensive Disorders of Pregnancy. Emerg Med Clin North Am. 2019;37:301-16.

4.

Phipps E, Prasanna D, Brima W, Jim B. Preeclampsia: Updates in Pathogenesis, Definitions, and Guidelines. Clin J Am Soc Nephrol. 2016;11:1102-13.

5.

Brosens I, Benagiano M, Puttemans P, D'Elios MM, Benagiano G. The placental bed vascular pathology revisited: a risk indicator for cardiovascular disease. J Matern Fetal Neonatal Med. 2019;32:1556-64. 6.

Bonagura TW, Pepe GJ, Enders AC, Albrecht ED. Suppression of extravillous trophoblast vascular endothelial growth factor expression and uterine spiral artery invasion by estrogen during early baboon pregnancy. Endocrinology. 2008;149:5078-87.

7.

Kumar S, Gordon GH, Abbott DH, Mishra JS. Androgens in maternal vascular and placental function: implications for preeclampsia pathogenesis. Reproduction. 2018;156:R155-67.

8.

Maliqueo M, Echiburu B, Crisosto N. Sex Steroids Modulate Uterine-Placental Vasculature: Implications for Obstetrics and Neonatal Outcomes. Front Physiol. 2016;7:152.

9 . 
Hertig A, Liere P, Chabbert-Buffet N, Fort J, Pianos A, Eychenne B, Cambourg A, Schumacher M, Berkane N, Lefevre $\mathrm{G}$, et al. Steroid profiling in preeclamptic women: evidence for aromatase deficiency. Am J Obstet Gynecol. 2010;203:477 e471-9.

10.

Berkane N, Liere P, Oudinet JP, Hertig A, Lefevre G, Pluchino N, Schumacher M, Chabbert-Buffet N. From Pregnancy to Preeclampsia: A Key Role for Estrogens. Endocr Rev. 2017;38:123-44.

11.

Thomopoulos C, Tsioufis C, Michalopoulou H, Makris T, Papademetriou V, Stefanadis C. Assisted reproductive technology and pregnancy-related hypertensive complications: a systematic review. J Hum Hypertens. 2013;27:148-57.

12.

Martin AS, Monsour M, Kawwass JF, Boulet SL, Kissin DM, Jamieson DJ. Risk of Preeclampsia in Pregnancies After Assisted Reproductive Technology and Ovarian Stimulation. Matern Child Health J. 2016;20:2050-6.

13.

von Versen-Hoynck F, Schaub AM, Chi YY, Chiu KH, Liu J, Lingis M, Stan Williams R, Rhoton-Vlasak A, Nichols WW, Fleischmann RR, et al. Increased Preeclampsia Risk and Reduced Aortic Compliance With In Vitro Fertilization Cycles in the Absence of a Corpus Luteum. Hypertension. 2019;73:640-9.

14.

Li Y, Zhao S, Yu Y, Ma C, Zheng Y, Niu Y, Wei D, Ma J. Risk factors associated with pre-eclampsia in pregnancies conceived by ART. Reprod Biomed Online 2019.

15.

Kolibianakis EM, Venetis CA, Tarlatzis BC. Role of the endocrine profile for the achievement of pregnancy with IVF. Reprod Biomed Online. 2009;18(Suppl 2):37-43.

16.

Tsai YR, Huang FJ, Lin PY, Kung FT, Lin YJ, Lin YC, Lan KC. Progesterone elevation on the day of human chorionic gonadotropin administration is not the only factor determining outcomes of in vitro fertilization. Fertil Steril. 2015;103:106-11.

17.

Imudia AN, Awonuga AO, Doyle JO, Kaimal AJ, Wright DL, Toth TL, Styer AK. Peak serum estradiol level during controlled ovarian hyperstimulation is associated with increased risk of small for gestational age and preeclampsia in singleton pregnancies after in vitro fertilization. Fertil Steril. 2012;97:1374-9.

18.

Royster GDt, Krishnamoorthy K, Csokmay JM, Yauger BJ, Chason RJ, DeCherney AH, Wolff EF, Hill MJ. Are intracytoplasmic sperm injection and high serum estradiol compounding risk factors for adverse obstetric outcomes in assisted reproductive technology? Fertil Steril. 2016;106:363-70 e363. 19.

Chen ZJ, Shi Y, Sun Y, Zhang B, Liang X, Cao Y, Yang J, Liu J, Wei D, Weng N, et al. Fresh versus Frozen Embryos for Infertility in the Polycystic Ovary Syndrome. N Engl J Med. 2016;375:523-33.

20. 
Maheshwari A, Pandey S, Amalraj Raja E, Shetty A, Hamilton M, Bhattacharya S. Is frozen embryo transfer better for mothers and babies? Can cumulative meta-analysis provide a definitive answer? Hum Reprod Update. 2018;24:35-58.

21.

Roque M, Haahr T, Geber S, Esteves SC, Humaidan P. Fresh versus elective frozen embryo transfer in IVF/ICSI cycles: a systematic review and meta-analysis of reproductive outcomes. Hum Reprod Update. 2019;25:2-14.

22.

Sha T, Yin X, Cheng W, Massey IY. Pregnancy-related complications and perinatal outcomes resulting from transfer of cryopreserved versus fresh embryos in vitro fertilization: a meta-analysis. Fertil Steril. 2018;109:330-42 e339.

23.

Ginstrom Ernstad E, Wennerholm UB, Khatibi A, Petzold M, Bergh C. Neonatal and maternal outcome after frozen embryo transfer: Increased risks in programmed cycles. Am J Obstet Gynecol. 2019;221:126 e121-6 e118.

24.

Lan KC, Huang FJ, Lin YC, Kung FT, Hsieh CH, Huang HW, Tan PH, Chang SY. The predictive value of using a combined Z-score and day 3 embryo morphology score in the assessment of embryo survival on day 5 . Hum Reprod. 2003;18:1299-306.

25.

Jobe SO, Tyler CT, Magness RR. Aberrant synthesis, metabolism, and plasma accumulation of circulating estrogens and estrogen metabolites in preeclampsia implications for vascular dysfunction. Hypertension. 2013;61:480-7.

26.

Salas SP, Marshall G, Gutierrez BL, Rosso P. Time course of maternal plasma volume and hormonal changes in women with preeclampsia or fetal growth restriction. Hypertension. 2006;47:203-8. 27.

Acikgoz S, Bayar UO, Can M, Guven B, Mungan G, Dogan S, Sumbuloglu V. Levels of oxidized LDL, estrogens, and progesterone in placenta tissues and serum paraoxonase activity in preeclampsia. Mediators Inflamm. 2013;2013:862982.

28.

Zhang Y, Wang T, Shen Y, Wang X, Baker PN, Zhao A. 2-Methoxyestradiol deficiency is strongly related to hypertension in early onset severe pre-eclampsia. Pregnancy Hypertens. 2014;4:215-9. 29.

Wan J, Hu Z, Zeng K, Yin Y, Zhao M, Chen M, Chen Q. The reduction in circulating levels of estrogen and progesterone in women with preeclampsia. Pregnancy Hypertens. 2018;11:18-25.

30 .

Shin YY, Jeong JS, Park MN, Lee JE, An SM, Cho WS, Kim SC, An BS, Lee KS. Regulation of steroid hormones in the placenta and serum of women with preeclampsia. Mol Med Rep. 2018;17:2681-8. 31. 
Csapo A. The luteo-placental shift, the guardian of pre-natal life. Postgrad Med J. 1969;45:57-64.

32.

Pijnenborg R, Anthony J, Davey DA, Rees A, Tiltman A, Vercruysse L, van Assche A. Placental bed spiral arteries in the hypertensive disorders of pregnancy. Br J Obstet Gynaecol. 1991;98:648-55.

33.

Johnson KM, Hacker MR, Resetkova N, O'Brien B, Modest AM. Risk of ischemic placental disease in fresh and frozen embryo transfer cycles. Fertil Steril. 2019;111:714-21.

34.

Devoto L, Henriquez S, Kohen P, Strauss JF. 3rd: The significance of estradiol metabolites in human corpus luteum physiology. Steroids. 2017;123:50-4.

35.

Jarvela IY, Pelkonen S, Uimari O, Makikallio K, Puukka K, Ruokonen A, Tekay A, Martikainen H. Controlled ovarian hyperstimulation leads to high progesterone and estradiol levels during early pregnancy. Hum Reprod. 2014;29:2393-401.

36.

Choux C, Carmignac V, Bruno C, Sagot P, Vaiman D, Fauque P. The placenta: phenotypic and epigenetic modifications induced by Assisted Reproductive Technologies throughout pregnancy. Clin Epigenetics. $2015 ; 7: 87$.

37.

Palomba S, Homburg R, Santagni S, La Sala GB, Orvieto R. Risk of adverse pregnancy and perinatal outcomes after high technology infertility treatment: a comprehensive systematic review. Reprod Biol Endocrinol. 2016;14:76.

38.

Milosevic-Stevanovic J, Krstic M, Radovic-Janosevic D, Stefanovic M, Antic V, Djordjevic I. Preeclampsia with and without intrauterine growth restriction-Two pathogenetically different entities? Hypertens Pregnancy. 2016;35:573-82.

39.

Howell KR, Powell TL. Effects of maternal obesity on placental function and fetal development. Reproduction. 2017;153:R97-108.

40.

Spradley FT. Metabolic abnormalities and obesity's impact on the risk for developing preeclampsia. Am J Physiol Regul Integr Comp Physiol. 2017;312:R5-12.

41.

Dayan N, Pilote L, Opatrny L, Basso O, Messerlian C, El-Messidi A, Daskalopoulou SS. Combined impact of high body mass index and in vitro fertilization on preeclampsia risk: a hospital-based cohort study. Obesity (Silver Spring). 2015;23:200-6.

42.

Hutcheon JA, Stephansson O, Cnattingius S, Bodnar LM, Wikstrom AK, Johansson K. Pregnancy Weight Gain Before Diagnosis and Risk of Preeclampsia: A Population-Based Cohort Study in Nulliparous Women. Hypertension. 2018;72:433-41. 
43.

Gaillard R, Durmus B, Hofman A, Mackenbach JP, Steegers EA, Jaddoe VW. Risk factors and outcomes of maternal obesity and excessive weight gain during pregnancy. Obesity (Silver Spring). 2013;21:1046-55.

\section{Tables}


Table1. Comparison of baseline and assisted reproductive technology (ART) cycle parameters between patients with a normal pregnancy and those who developed preeclampsia.

\begin{tabular}{lll}
\hline Characteristic & Normal pregnancy & $P$ Preeclampsia. \\
\cline { 2 - 4 } & $(\mathrm{n}-594)$ & $(\mathrm{n}=28)$
\end{tabular}

\begin{tabular}{|c|c|c|c|}
\hline Age (y) & $34.0(5.4)$ & $35.0(4.8)$ & NS \\
\hline Husband age (Y) & $36.0(6.5)$ & $37.5(5.8)$ & NS \\
\hline $\operatorname{BMI}\left(\mathrm{kg} / \mathrm{m}^{2}\right)$ & $22.0(4.3)$ & $24.0(8.0)$ & NS \\
\hline Body height(cm) & $160.0(7.0)$ & $159.0(22.0)$ & NS \\
\hline Body weight(kg) & $56.0(10.0)$ & $62(22.0)$ & NS \\
\hline Body weight at delivery $(\mathrm{kg})$ & $70.0(12.4)$ & $80.5(14.4)$ & $<0.001$ \\
\hline Gestational weight gain $(\mathrm{kg})$ & $13.0(7.0)$ & $19.6(14.3)$ & 0.002 \\
\hline Primary infertility & $58.80 \%$ & $46.40 \%$ & NS \\
\hline
\end{tabular}

\begin{tabular}{lcc}
\hline tubal factor & $27.40 \%$ & $21.40 \%$ \\
\hline ovulatory factor & $12.20 \%$ & $10.70 \%$ \\
\hline endometriosis factor & $13.20 \%$ & $21.40 \%$ \\
\hline male factor & $27.70 \%$ & $14.30 \%$ \\
\hline other and combine factors & $19.50 \%$ & $29.50 \%$
\end{tabular}

Protocol

\begin{tabular}{lcc}
\hline antagonist & $40.00 \%$ & $32.10 \%$ \\
\hline agonist & $60.00 \%$ & $67 . .9 \%$ \\
\hline Days of stimulation & $9.0(2.0)$ & $9.0(2.0)$ \\
& & NS \\
\hline Total gonadotropins administered (Ampoules of 75 IU FSH) & $29.0(12.0)$ & $31.0(10.8)$ \\
\hline Endometrium thickness $(\mathrm{cm})$ & $1.4(0.4)$ & $1.3(0.5)$
\end{tabular}


Continuous data are expressed as median (interquartile range) and dichotomous data, a percentage of the population.

BMI : body mass index

ICSI: intracytoplasmic sperm injection.

NS: not significant 
Table2. Comparison of obstetric and perinatal outcomes between patients with a normal pregnancy and those who developed preeclampsia.

\begin{tabular}{|c|c|c|c|}
\hline \multirow[t]{2}{*}{ Characteristic } & Normal pregnancy & preeclampsia. & \multirow[t]{2}{*}{$P$-value } \\
\hline & $(n-594)$ & $(n=28)$ & \\
\hline Multiple pregnancy & $31.90 \%$ & $32.10 \%$ & NS \\
\hline CS & $53.50 \%$ & $82.10 \%$ & $<0.001$ \\
\hline Sex ratio(male/female) & $1.07(406 / 381)$ & $1.31(21 / 16)$ & NS \\
\hline \multirow[t]{2}{*}{ Intrauterine growth restriction } & $27.78 \%$ & $32.14 \%$ & NS \\
\hline & & & $<0.001$ \\
\hline GA(single) & $38(2)$ & $36(4)$ & \\
\hline \multirow[t]{2}{*}{ Birth weight (single) } & & & NS \\
\hline & $3100(530)$ & 2910(1690) & \\
\hline \multirow[t]{2}{*}{ GA (twin) } & & & NS \\
\hline & $36(3)$ & $36(3)$ & \\
\hline \multirow[t]{2}{*}{ Birth weight (Twin) } & & & NS \\
\hline & $2370(667)$ & $2420(485)$ & \\
\hline
\end{tabular}

Continuous data are expressed as median (interquartile range) and dichotomous data, a percentage of the population.

CS: Cesarean section

GA: gestational age

NS: not significant 
Logistic analysis of factors related to preeclampsia

Univariable analysis $\quad$ Multivariable analysis

e Crude odds ratio(95\%CI) $\quad P$ value $\quad$ Adjusted odds ratio(95\%CI) $P$ value

$34 \mathrm{vs}>34) \quad 2.205(1.015,4.792) \quad 0.046 \quad 3.762(0.444,14.510) \quad$ NS

$\left.\mathrm{r} / \mathrm{m}^{2}\right)(\leq 22 \mathrm{vs}>22) \quad 1.484(0.650,3.388) \quad$ NS $\quad 0.293(0.019,4.506) \quad$ NS

SI $\quad 0.280(0.083,0.939) \quad 0.039 \quad 0.622(0.094,4.138) \quad$ NS

$\mathrm{mL})(>1.2 \mathrm{vs} \leq 1.2) \quad 2.095(0.841,5.221) \quad$ NS $\quad 2.645(0.482,26.080) \quad$ NS

$\mathrm{k}(\mathrm{pg} / \mathrm{mL})(<1200 \mathrm{vs}>1200) 2.285(1.002,5.214) \quad 0.044 \quad 4.634(1.061,20.222) \quad 0.041$

onal weight gain $(\mathrm{kg})$

$\mathrm{s} \leq 20) \quad 8.475(3.188,22.531) \quad<0.001 \quad 13.601(3.784,48.880) \quad<0.001$

$\mathrm{CI}=$ confidence interval; $\mathrm{OR}=$ odds ratio. 